\title{
Analysis of Fire Suppression Effectiveness Using a Physically Based Computer Simulation
}

\author{
L. M. PIETRZAK and G. A. JOHANSON \\ Mission Research Corporation \\ P.O. Drawer 719 \\ Santa Barbara, California 93102, USA
}

\section{ABSTRACT}

The Swedish Fire Research Board and the U.S. Federal Emergency Management Agency are sponsoring a project to further the understanding of the basic mechanisms involved, as well as to support the development of standards for and to seek ways of improving the performance of portable fire suppression systems used by fire departments. This effort includes both experiments and computer model development work.

This paper describes a physically based computer model developed to simulate one aspect of the problem: the manual suppression of post-flashover fires. This includes: (1) a discussion of the physical basis behind the model; (2) a comparison of model predictions with available experimental data; and (3) an analysis of fire suppression effectiveness using the model.

The analysis concludes that, when direct assess and extinguishment of the burning fuel is not possible, improved fire control occurs with water sprays having a Rosin-Rammler distribution of droplet sizes and volume medium drop diameters in the 0.15 to $0.35 \mathrm{~mm}$ range. This agrees with available experimental data. It is also shown that firefighting venting and standoff distance requirements may lead to more severe fires requiring more water for control. Finally, the analysis shows that venting and water spray induced air/gas flow effects also serve to channel hot steam and gases away from the fire-fighter adding to his safety. Additional experimental work is also recommended before all these conclusions can be considered definitive.

\section{INTRODUCTION}

The focus of the effort described in this paper is on the suppression of post-flashover or fully developed compartment fires using manually applied water spray. The paper begins by describing a fire suppression computer simulation developed as part of the research effort. This includes inputs, outputs, and physical effects and interactions modeled, both with and without suppression effects. Example model results and sensitivities for a single compartment geometry are then described including comparisons with available experimental data.

\section{MODEL. DESCRIPTION}

The fire suppression model described here is called the Fire Demand (FD) Model. Refs. 1 through 6 describe this FD Model and its applications in detail. The following briefly summarizes the physical effects and interactions incorporated in the FD Model. 
Previous investigators (Refs. $7,8,9$ ) successfully modeled the post-flashover fire in its freely burning phase (without suppression). In the FD Model, the freely burning segment of the fire history follows their methods and calculates the fire development in time in terms of lumped parameters describing the energy and mass balance of the compartment as a whole. The FD Model adds the effect of water suppression application to this work.

The FD Model is capable of simulating fires involving both char forming and non-charring solid fuels in compartments with single or multiple vents of different sizes and in different locations, and venting changes with time due to firefighting activities, including water spray induced air inflow.

In the freely burning period (without suppression effects), the fire behavior is determined by the room itself (dimensions, size, and shape of the ventilation openings, thickness and composition of bounding walls, etc.) and by fuel features (heat of combustion, weight of fuel, total surface area of fuel, etc.). The fire behavior is described by the average temperature of the room gas, the average temperature of the walls and ceilings, floor temperature, the retained heat in the room, and the burning rate of the fuel. The fundamental basis of the freely burning post-flashover fire model is a mass balance and a heat balance of the gas contained in the compartment. One key operational feature of the FD Model is the causal relation whereby the buoyancy of the hot room gas drives combustion products out of the ventilation opening and draws fresh air in. In turn, the rate of fresh air entry determines (for this post-flashover case) the combustion rate, which is the major source of heat for the room gas.

The factor of water application modifies the fire behavior drastically. The cooling of interior gases and interior surfaces by water vaporization, the choking of ventilation by the exit of steam, and the direct extinguishment of burning surfaces reachable by water are simulated by the FD Model. The FD Model also accounts for any additional air from outside being forced into the fire by the induced effects of the water spray. The relative magnitude of these effects determines whether fire control is achieved (with sufficient water) or whether the fire only stabilizes at lower temperatures (with insufficient water). To estimate these effects the FD Model requires the specification of the time of water application, the distance of the hose-nozzle from the vent, and water spray characteristics such as: the flow rate, pressure, the distribution and volume medium diameter of water drops in the spray pattern, the cone angle of the stream, the sweep time required for the stream to cover the interior of the compartment, and the fraction of fuel area accessible to water impact. Apart from the fire conditions--as determined by the compartment and fuel--these are the factors which determine the FD Model estimate of suppression effectiveness.

The level of physical detail incorporated into the FD Model is determined by its practical objectives and the desire for simplicity and computability. The suppression effects are accounted for using relatively simple submodels consistant with the lumped parameter nature of the overall model. These submodels are based on the overall assumption that on introduction of the water spary into a fully involved fire, the resulting steam expands and mixes rapidly with the compartment gases so that one can continue to represent the processes in terms of Tumped parameters characterized by average temperatures and heat fluxes within the compartment.

Central to the estimation of water effects is the apportionment of the water volume into three parts: (1) a part which is blown away thorugh failure to penetrate the updrafts in the compartment, (2) a part which is vaporized in the compartment gas, and (3) the remainder which impacts the fuel and interior surfaces in liquid form. This simplified submodel assumes a water drop of given 
initial diameter falls and evaporates in a compartment characterized by a uniform temperature and a uniform updraft velocity. The temperature is the gas temperature of the compartment and the updraft velocity is estimated from the room geometry and the air circulation rate in the compartment. The water drop is assumed to fall vertically at terminal velocity (relative to the gas) determined by its instantaneous diameter which changes as the drop falls. For given compartment conditions there are two critical drop diameters: the diameter of a drop whose terminal velocity equals the updraft velocity, and the diameter of a drop which will just reach the floor before its diameter has decreased by evaporation to a size small enough to be swept away by the updraft. Results for single drops are averaged over an assumed drop size distribution to produce a water partitioning. The model assumes a Rosin-Rammler (Ref. 10) distribution of drop sizes which may be completely characterized by the volume median drop diameter, half of the water volume occurs in drops below this size and half above. No account is taken of any further breakup of the spray by impact on surfaces. The fraction of water which is vaporized cools the compartment gas. The fraction which reaches the floor is re-interpreted as the fraction which reaches interior surfaces and fuel, and is distributed to them in proportion to wall/ceiling area, floor area, and exposed fuel surface area.

Regarding the cooling of hot, non-burning interior surfaces, account is taken of the fact that only a fraction of these surfaces are instantaneously impacted at any one time by a sweeping water spray of limited cone angle. There is therefore a residence time during which cooling of the surface can occur. In the case of walls the fraction vaporized there and the fraction which runs off is estimated. In estimating surface cooling the impacting water spray is assumed to coalesce into a thin sheet over the impacted surface. The average rate of heat extraction is then calculated as the limiting value obtained by either the amount of water available or by conduction from the interior. If conduction 1 imits, the surface temperature under hose impact is assumed to equal $100^{\circ} \mathrm{C}$ and the vaporized and runoff water fraction is calculated based on this. If the avallable water is limiting all the water is assumed to vaporize and the surface temperature is calculated accounting for the cooling effect of this water and the residence time of the hose stream. For the water reaching and standing on the floor, another limiting condition accounted for is the rate of heat transfer possible by boiling.

The fraction of the total burning fuel surface area accessible to water impact may also vaporize liquid water and thereby reduce the rate of heat generation. Extinguishment of this fuel area occurs by different criteria depending upon the type of fuel. For charring cellulosic or plastic fuels, extinguishment is assumed to occur when the rate of heat extraction by water vaporization exceeds the heat generation rate by charring combustion alone.

For non-charring fuels, the extinguishment submodel follows the conditions examined experimentally by Magee and Reitz (Ref. 11) wherein critical water application rates were measured as a function of incident radiation. The model assumes that for post-flashover conditions, radiation from the fire plumes, hot surfaces and gases in the compartment control the rate of fuel pyrolysis or vaporization even as a portion of the fuel is directiy extinguished by the water. The critical water application rate is therefore taken to be that required to counter the heat received by radiation-i.e., extinguishment is 
assumed to occur when the rate of heat extraction by water vaporization exceeds the net heating rate to the exposed fuel by radiation.*

The rate of heat extraction is calulated for the following two cases: For non-charring fuel surfaces burning in a rigid or softened state, the impacting water spray is assumed to coalesce into a thin sheet and to act as a thermal radiation barrier from above and a coolant that cools the hot fuel from below. For surfaces burning in a molten or liquid state the impacting water is assumed to penetrate the surface and cool the fuel from within. Emperical data from Reference 11 is used to account for possible burning rate enhancement due to splashing of droplets on impact and/or bubbling of the vaporizing water from within.

\section{COMPARISON WITH EXPERIMENTS}

Results from the FD Model compare favorably with the limited experimental data available which addresses the suppression of post-flashover fires using manually applied water spray (Refs. 13 through 18).

For the fire conditions examined in Ref. 13, the experiments found that water application rates greater than $25 \mathrm{l} / \mathrm{min}$ were required for fire control. The FD Model predicts application rates for fire control of $34 \mathrm{l} / \mathrm{min}$ to $57 \mathrm{l} / \mathrm{min}$ depending on the volume medium drop size. In Ref. 14, the amount of water vaporized was also measured. For the experimental conditions tested, approximately 94 to 110 \& were required. The FD Model predicts 64 to 130 \& again depending on the volume medium drop size. Although the above results compare favorably, the experimental data in Refs. 13 and 14 do not record the values of several parameters (e.g. drop sizes) to which model results are sensitive, so reasonable parametric values were chosen for the simulation (Ref. 1). Further experimental calibration and validation of the FD Model is required.

In addition to the above, a series of fully envolved fire suppression experiments using water sprays were recently completed in osaka, Japan (Refs. 15 and 16). These tests were conducted in compartments characteristic of residential occupancies. The objective of the Japanese tests was to establish the sizes of water droplets which controlled the fire with minimum water runoff and damage. A major conclusion of this work was that average droplet sizes in the range of 0.2 to $0.3 \mathrm{~mm}$ achieved the best results. These droplet sizes were obtained from nozzles operating under a pressure of $10 \mathrm{~kg} / \mathrm{cm}^{2}$ with a discharge of $180 \mathrm{l} / \mathrm{min}$. The nozzle produces fine droplet sizes without resorting to high discharge pressures. The nozzle design diverts part of the water through a whirler to produce a flowing vortex of water. The water in the vortex is then mixed with a high velocity stream of the remaining water and ejected from the nozzle outlet. The nozzle is also capable of straight stream application. Unfortunately the information regarding vent size and other compartment characteristics reported in Refs. 15 and 16 is insufficient to allow direct comparison with the FD Model. Furthermore, these papers do not define specifically what is meant by "average" droplet size or how it was measured. Although the optimal range of 0.2 to $0.3 \mathrm{~mm}$ appears to agrees with FD Model predictions as presented in the next section, definitive comparisons are not possible until the above uncertanties are resolved.

*For the case of suppression of small fires consisting of a single fire plume or multiple but non-interacting plumes, convection or conduction from the local plume rather than radiation can dominate. In Ref. 12, Rasbash calculates the critical rate at which a water spray abstracts heat from liquid fuels at the surface to reduce the vaporization below fire sustaining levels. 
In addition to the above, it has recently come to the author's attention that a series of post-flashover fire suppression experiments were carried out in England in the later 1950's (Refs. 17, 18 and 19). Further work is required to adjust the input parameters of the FD Model to allow comparisons to these tests.

\section{EXAMPLE MODEL SENSITIVITIES}

The results presented in what follows uses the Rosin-Rammler distribution of drop sizes measured for sprinkler heads (Ref. 10). In the future, a more thorough analysis is required using actual measured flow rates and spray characteristics from currently used manual hose-nozzle equipment as well as relevant data reported in Refs. 18, and 20 through 24 .

FD Model results and principal sensitivities are presented in terms of graphs that relate water application rate per unit total interior area of the room to the volume median drop diameter of the water spray. For example, for the room and fuel conditions listed in Table 1, simulation predictions of total water requirements are given in Fig. 1.

Table 1. Input conditions used in the example for charring fuels in a compartment having a single vent.

\section{COMPARTMENT DESCRIPTION}

Room Height

Floor Area

Area of Walls and Ceiling

Window Area

Window Height

Wall/Ceiling/Floor Thickness

Wa11/Ceiling/Floor Conductivity

Wall/Ceiling/Floor Specific Heat

\section{FUEL DESCRIPTION}

\section{Fuel Type}

FueT Load

Fuet Surface Area

Fuel Surface Area Exposed to Water

Effective Heat of Combustion

\section{WATER APPLICATION DESCRIPTION}

Distance of Nozzle from Vent

Time of Water Application

After Flashover

Nozzle Pressure

Cone Angle of Hose Stream

Sweep Time to Cover Compartment

Volume Median Water Drop Diameter*

Flow Rate of Water
INPUT VALUE

$$
\begin{aligned}
& 2.44 \mathrm{~m}^{2} \\
& 11.11 \mathrm{~m}^{2} \\
& 42.85 \mathrm{~m}^{2} \\
& 1.69 \mathrm{~m}^{2} \\
& 1.12 \mathrm{~m} \\
& 0.15 \mathrm{~m} \\
& 0.00833 \mathrm{kca} / / \mathrm{m} / \mathrm{min} /{ }^{\circ} \mathrm{C} \\
& 250 \mathrm{kca} 7 / \mathrm{m}^{3} /{ }^{\circ} \mathrm{C}
\end{aligned}
$$

\section{Wood}

$21.95 \mathrm{~kg} / \mathrm{m}^{2}$

$28.52 \mathrm{~m}^{2}$

$0 \%$

$2575 \mathrm{KcaT} / \mathrm{Kg}$

$0 \mathrm{~m}$

5 minutes

$1 \mathrm{Kg} / \mathrm{cm}^{2}$

$60^{\circ} \mathrm{C}$

5 seconds

Varies

Varies

*Assuming a Rosin-Rammler drop size distribution (Ref. 10). 


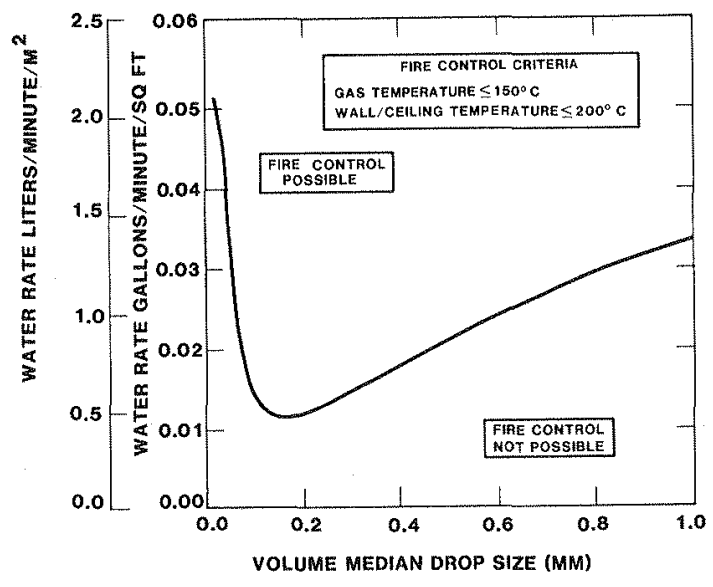

Fig. 1. Example results for charring wood fuels at $0 \%$ exposed fuel fraction.

The "control-failure" line appearing in Fig. 1 divides the graph into two regions characterized by combinations of water delivery rate and volume median drop diameters which are successful or unsuccessful in controlling the fire. The gas and surface temperatures which the model employs as fire control criteria are arbitrarily selected and results are sensitive to them (Refs. 1 and 2).

Note, in Fig. 1, that as the volume median drop diameters increase from about $0.05 \mathrm{~mm}$ to 1 $\mathrm{mm}$, the bounding control failure line first slopes downward, achieving a minimum at about $0.15 \mathrm{~mm}$, and then increases with drop size. This behavior reflects the sensitivity to drop size of fires controlled primarily by gaseous and non-burning surface cooling as we11 as vent choking mechanisms. For the example shown in Fig. 1, this means a significant fraction of the drops with sizes less than $0.15 \mathrm{~mm}$ are blown away by hot gases and are not effective in achieving fire control. On the other hand, droplets greater than $0.15 \mathrm{~mm}$ result in a significant fraction that fail to fully vaporize and, therefore, contribute to water runoff. In each case water is wasted and, because of this, the total water application rates required for fire control increases.

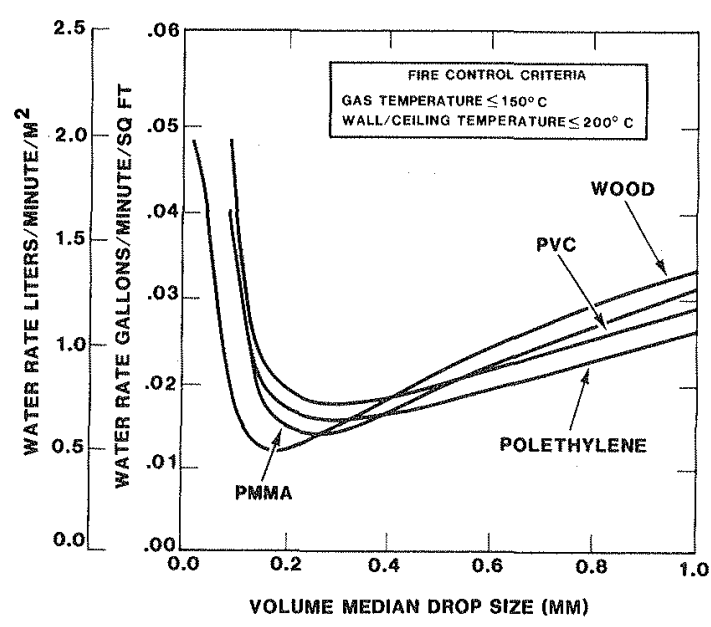

Fig. 2. Effect of fuel type (exposed fuel fraction $=0 \%$.
The previous results are for cellulosic fuels which form a char layer while burning. Fig. 2 presents results for three different non-charring plastic fuels compared to the wood case. Other than fuel properties, the compartment and water delivery characteristics are the same as for the wood case.

Unlike fuels with an oxidizing char layer, the mass loss rate due to the pyrolysis or vaporization of fuels that burn without developing an oxidizing char layer depends strongly on the degree of thermal heat transfer to the fuel bed from the hot or burning gases and surfaces in the compartment. For these materials, fuel-thermal coupling effects have also been modeled. In general, one can see from Fig. 2 that for the specific compartment examined the "failurecontrol" line tracts fairly closely for all the fuels examined. 


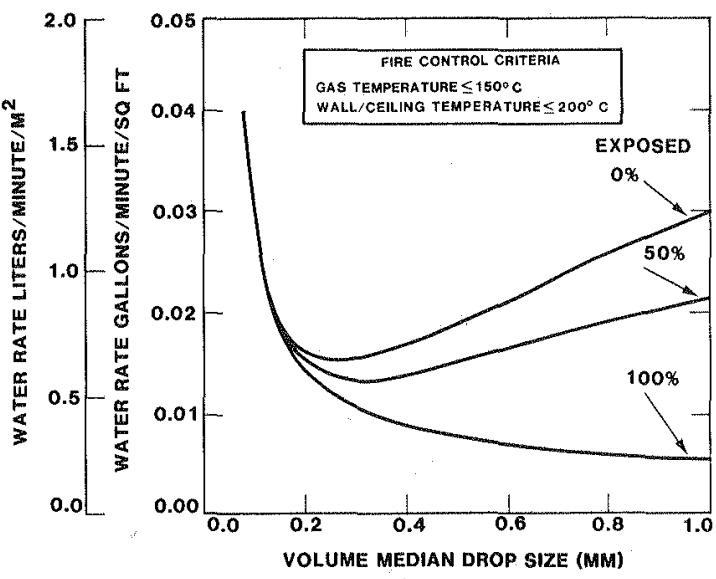

Fig. 3. Sensitivity to water exposed fuel fraction--PMMA fue?.
Fig. 3 illustrates the effect of the "exposed fuel fraction". In general, the shape of the line defining the control-failure region is sensitive to the fraction of the burning fuel area accessible to direct water impact. If little or no fuel can be directly extinguished, control occurs by gaseous/surface cooling and vent choking. The gaseous cooling, which is a very sensitive function of drop size, produces the sharp minimum of the curve at the optimum drop size. If the exposed fuel fraction is high, direct extinguishment rather than gaseous/non-burning-surface cooling and vent choking dominate. For this case, the critical application rate required for fire control is similar at the lower drop sizes but after a certain point continues to decrease as drop size increases. Here fire control is effected by a decrease of heat production rate consequent to extinguishment of burning surfaces. The larger drops become more effective since they penetrate better, loose less of their volume by evaporation and carry more water to the burning surfaces. This means that if the seat of the fire is directly accessible, in order to minimize water usage, extinguishment is preferred to gaseous/non-burning surface cooling and vent choking. On the other hand, because of standoff requirements for safety, direct access is not always possible in post-flashover fire situations.

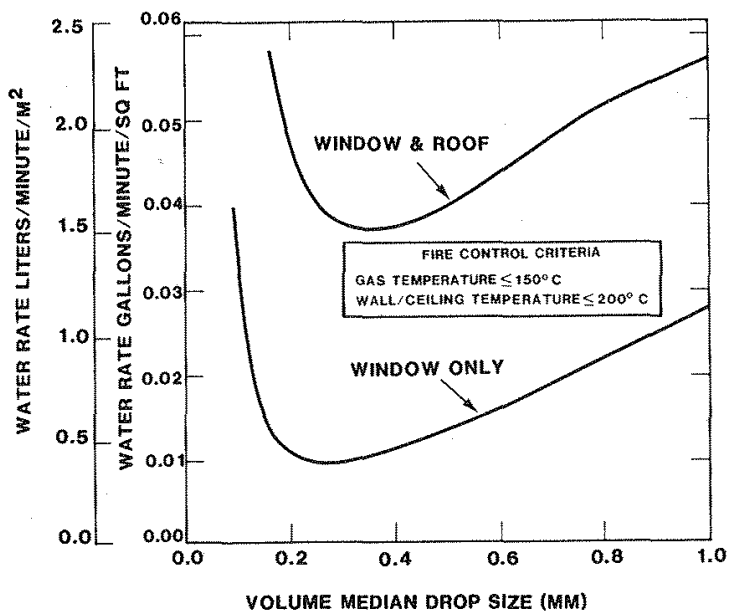

The previous results are for a compartment having a single vent where the hose-nozzle is positioned within the open vent of the compartment. The FD Model can a) so handle more generalized vent ing conditions. This includes multiple wall and/or roof vents of different sizes and location and opened at different times. This also includes the opening of wall or roof vents occurring as part of the firefighting venting operations as well as water spray induced air inflow for hosenozzles positioned away from the vent.

Fig. 4 shows the effect of a firefighter opening a 1 square meter hole in the roof of the compartment beginning one minute before water is applied and proceeding at a rate of $1 \mathrm{~m}^{2} / \mathrm{min}$. 
Except for the added roof vent, the other compartment and water delivery characteristics are the same as given in Table 1 . It is clear from Fig. 4 that venting has a significant effect on the flow-rates and drop sizes required to control the fire.

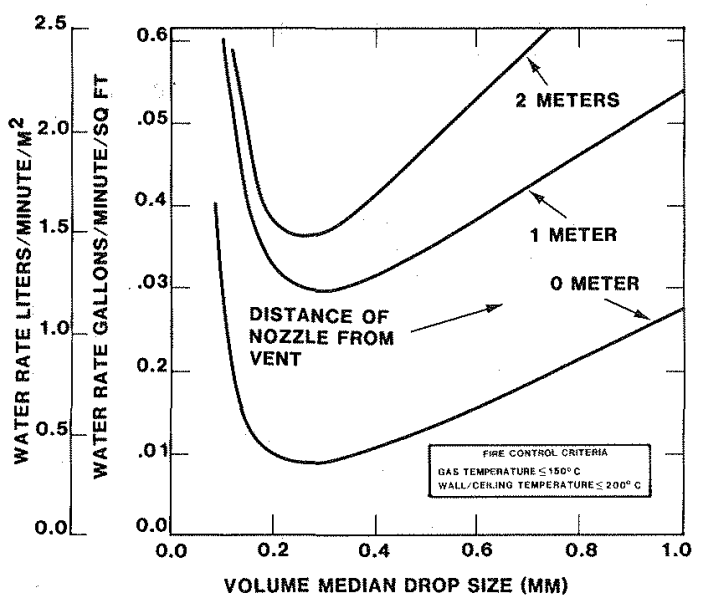

Fig. 5. Effect of water spray induced air inflow (PMMA- - 0\% exposed fuel fraction).

Fig. 5 shows the significant effect induced air inflow from the water spray has on the results for nozzles positioned away from the vent a distance of respectively, zero, $1 \mathrm{~m}$ and $2 \mathrm{~m}$. A single vent $(1.12 \mathrm{~m} \times 1.509 \mathrm{~m})$ with PMMA fuel is used in this example. Fig. 5 applies to nozzle pressures as low as $15 \mathrm{psi}$ to as high as 100 psi-ui.e. essentially the same curves are obtained for each. An analysis of these results shows that after a certain level of induced air inflow, the burning and intensity of the fire becomes limited by the amount of available fuel rather than air. This Timit is apparently exceeded by the air inflow generated by 100 psi as well as 15 psi for the water flow rates indicated in Fig. 5. In general, Fig. 5 shows the importance of positioning the nozzle as close as possible to the vent consistent with standoff distance requirements for safety. This minimizes water spray air induced effects reducing the water flow rates required by factors of three or more.

\section{CONCLUSIONS}

In general, the above results and those reported in Ref. 1 through 4 indicate that improved fire control is possible with water sprays having a Rosin-Rammler distribution of droplet sizes and volume median drop diameters in the 0.15 to $0.35 \mathrm{~mm}$ range. This optimal range applies only when direct assess and extinguishment of the burning fuel is not possible. This range also implies no further breakup of the water jet or spray by impact on solid surfaces. A more thorough analysis using actual measured manual hose-nozzle spray, a broader range of compartment and fuel characteristics and additional experimental verification work is required before these conclusions $c$ an be considered definitive.

The FD Model results also suggest that firefighting venting and standoff distance requirements can lead to more severe fires requiring more water for control. They also suggest that venting together with the enhanced gas/air velocities from water spray induced effects also serve to channel the hot steam and products of combustion away from the firefighter and therefore have important safety implications quite apart from fire control. More analysis and experimental work is required to understand the tradeoffs and identify if there is perhaps some better balance between, for example, fireground venting or similar activities and the resulting water spray requirements. This can help establish important previously unavailable quantitative rules of thumb to follow on the fireground as well as improvements in firefighting equipment performance. 


\section{REFERENCES}

1. Pietrzak, L.M., Johanson, G.A., Ball, J.A. A Physically Based Fire Suppression Computer Simulation For Post-Flashover Compartment Fires -Applications, Experimental Requirements, Software Documentation and User's Guide, Mission Research Corporation Report MRC-R-846, 1985.

2. Pietrzak, L.M., and Ball, J.A., "Investigation to Improve the Effectiveness of Water in the Suppression of Compartment Fires," Fire Research, 1, 291-300, 1978.

3. Pietrzak, L.M. and Bal1, J.A., "Optimizing the Mobility and Fire Suppression Performance of Fire Engines Using Physically Based Computer Simulations," Fou $-\mathrm{Br}$ and, 1979.

4. Pietrzak, L.M., and Patterson, W.J., "Effect of Nozzles on Fires Studied in Terms of Flow Rate, Droplet Size," Fire Engineering, Vol. 132, No. 12, 1979.

5. Pietrzak, L.M., and Ball, J.A., "A Physically Based Fire Suppression Computer Simulation--Definition, Feasibility Assessment, Development Plan and Applications," MRC-R-732, Mission Research Corporation, 1983.

6. Pietrzak, L.M., et al., "Decision Related Research on Equipment Technology Utilized by Local Government: Fire Suppression--Phase II Research Report," National Science Foundation Report No. NSF/RA-770207, 1977.

7. Kawagoe, K., "Fire Behavior in Rooms," Building Research Institute Report No. 27, Tokyo, 1950.

8. Kawagoe, K., "Estimation of Fire Temperature Time Curve in Rooms," Building Research Paper No. 29, Buflding Research Institute, Tokyo, 1967.

9. Barbaruskas, V., and Williamson, R.B., "Post-Flashover Compartment Fires, "Report No. USBFRG 75-1, Fire Research Group, University of California, Berkeley, 1975.

10. Rosin, P. and Rammler, E.J., I. Institute Fuel, I, 1933.

11. Magee, R.S., and Reitz, R.D., "Extinguishment of Radiation Augmented Plastic Fires by Water Sprays," Factory Mutual Research, FMRC Serial No. 22357-1, 1974 .

12. Rasbash, D.J., "The Extinction of Fires by Water Spray," Fire Research Abstracts and Review, Vol. 4 (1 \& 2), 28-53, 1962.

13. Salzberg, F., Vodvarka, F.J., and Maatman, G.C., "Min imum Water Requirements for Suppression of Room Fires," Fire Technology, 6 (1), 221970.

14. Fuchs, P., "Brand-und Loschversuche Mit Vershiedenen Loschmitteln in Einem Versuchsraum Naturlicher Gross," Fire Protection Seminar, Karsruhe, Germany, VFDB, 1976.

15. Osuga, Ichinosuke, "Development of a Fog Stream-Fire Extinguishing System for Medium and High Storied Buildings," OSAKA, No. 4, 1983.

16. "Osaka Fire Department Fights Water Damage," Urban Innovation Abroad, 1984. 
17. Pickard, R. W., Hird, D., et a1., "Use of High and Low Pressure Water Spray Against Fully Involved Room Fires," Fire Research Station Note 388, England, 1959.

18. Nichols, S. and Freeman, S., D"etermination of Drop Sizes of High and Low Pressure Water Sprays, "Fire Research Station Note 373, England, 1959.

19. Thomas, P. H. and Smart, P.M., "Fire Extinction Tests in Rooms," Fire Research Station Note No. 121, England, 1954.

20. Rasbash, D. J., "The Properties of Sparys Produced by Batteries of Impinging Jets, "Fire Research Station Note No. 181, England, 1955.

21. Rasbash, D. J. and Stark, G. W. V., "Design of Sprays for Protective Installations, "Part III, Fire Research Station Note No. 303, 1958.

22. Fry, J.F. and Smart, P. M. T., "The Production of Water Sprays for Fire Extinction," Quart. Inst. Fire Engrs. Edinb., 13 (10) 82-104, 1953.

23. "Characteristics of water spray nozzles including those designated as fog nozzles for fire-fighting use," National Board of Fire Underwriters, Chicago, 1944.

24. Mobius, K., "Experience Obtained with the Testing of Spray Nozzles," V.F.D.B., 5 (2) $33-42,1956$. 